\title{
Embolización de trasplante renal no funcionante: experiencia inicial
}

\author{
Non-functioning renal transplant embolization: initial experience
}

Sebastián Amicone ${ }^{1}$, Marcelo Ventre ${ }^{1}$, Hugo Paggi ${ }^{1}$, Carlos Hernández ${ }^{1}$, Nicolás Nitti ${ }^{1}$, Darío Cavalie ${ }^{1}$, Fabián Taylor ${ }^{1}$, María Bayón ${ }^{1}$

\section{RESUMEN}

Hasta un $40 \%$ de los pacientes que vuelven a diálisis tras un trasplante renal fallido desarrollan intolerancia inmunológica al injerto cuando se suspende o disminuye la inmunosupresión. La reintroducción de la terapia inmunosupresora conlleva elevado riesgo de infecciones, neoplasias y riesgo cardiovascular. La trasplantectomía está asociada a una considerable morbimortalidad. La embolización del trasplante renal no funcionante constituye una alternativa segura y eficaz para el tratamiento de estos pacientes. Se presenta la experiencia inicial de un servicio de Hemodinamia polivalente de la ciudad de La Plata.

Palabras claves: embolización terapéutica, trasplante renal, síndrome de intolerancia.

\begin{abstract}
Up to $40 \%$ of patients who return to dialysis after a failed renal transplant develop immunologic intolerance to the graft when immunosuppression is suspended or reduced. The reintroduction of immunosuppressive therapy carries a high risk of infections, neoplasms and cardiovascular risk. Transplantectomy is associated with considerable morbidity and mortality. The embolization of the non-functioning renal transplant constitutes a safe and effective alternative for the treatment of these patients. We present the initial experience of a polyvalent hemodynamic service in the city of La Plata.
\end{abstract}

Key words: therapeutic embolization, kidney transplantation, failed kidney graft.

Revista Argentina de Cardioangiología Intervencionista 2019;10(4):0179-0183. https://doi.org/10.30567/RACI/201904/0179-0183

\section{INTRODUCCIÓN}

La enfermedad renal crónica (ERC) es un problema de salud pública mundial. La manifestación más grave de la enfermedad renal es la insuficiencia renal crónica terminal (IRCT), que lleva al paciente a la necesidad de terapia de sustitución renal con diálisis crónica o trasplante renal. La IRCT se ha duplicado en los últimos 10 años y se encuentra en aumento el número de pacientes en tratamiento con diálisis, así como el número de trasplantes renales. Es de prever en Argentina un aumento progresivo del número de pacientes que reciban un trasplante renal ${ }^{1}$. Un porcentaje significativo de pacientes vuelven a requerir diálisis tras el fallo del trasplante. Hasta un $40 \%$ de los pacientes que vuelven a diálisis tras un trasplante renal fallido desarrollan intolerancia inmunológica al injerto cuando se suspende o disminuye la inmunosupresión ${ }^{2}$. La reintroducción de la terapia inmunosupresora conlleva elevado riesgo de infecciones, neoplasias y aumento del riesgo cardiovascular ${ }^{3}$. La trasplantectomía está asociada a una considerable morbimortalidad ${ }^{4}$. La embolización del trasplante renal no funcionante constituye una alternativa segura y eficaz para el tratamiento de estos pacientes.

\section{MATERIALES Y MÉTODOS}

Se realiza un análisis descriptivo de una serie de pacientes con síndrome de intolerancia luego de trasplante renal fallido tratados mediante embolización en el Hospital San Martín de La Plata. El Servicio de Trasplante de nuestro hospital realiza un promedio de 50 trasplantes renales por año; des-

1. Hospital Interzonal General de Agudos San Martín, La Plata

$\triangle$ Correspondencia: Dr. Sebastián Amicone. Calle 7, No 1244, Dto 5. La Plato. 0221-155658112,0221-4838497

Los autores no declaran conflictos de intereses

Recibido:06/6/2019|Aceptado:04/09/2019 de el año 1996 se han realizado 885, el 10\% de estos a partir de donante vivo y el resto, cadavérico. Las causas por las que más frecuentemente se llega al trasplante son: insuficiencia renal crónica idiopática 34,8\%, glomerulopatías 20\%, nefroangioesclerosis $11,07 \%$, poliquistosis renal $9,04 \%$ y diabetes $3,16 \%$. Los pacientes son procedentes principalmente de ciudades del área correspondiente al CRAI SUR, que corresponde al sur de la provincia de Buenos Aires. Del total de pacientes trasplantados, el 24,75\% (219) reingresó a diálisis (luego de períodos variables desde el trasplante) en los 23 años de nuestra experiencia. Aproximadamente el 40\% de estos pacientes presenta síndrome de intolerancia renal. Han sido tratados a lo largo de los años con las distintas alternativas disponibles: tratamiento inmunosupresor, trasplantectomía y en los últimos años, embolización (Figura 1). La embolización se realiza en la Sala de Hemodinamia. Se utiliza anestesia local, se realiza punción de arteria femoral homolateral al injerto y se coloca introductor de 5 French. Se administran 5000 UI de heparina sódica intraarterial. Se cateteriza selectivamente arteria renal del injerto con catéter de 5 Fr (Cobra o Simmons 1). Se realiza embolización con microesferas de polivinilo 300-500 micras contenidas en jeringa prellenada diluidas con solución fisiológica y contraste. De ser necesario y técnicamente posible, se realiza embolización de arteria renal del injerto con coils fibrados. Se retira introductor en sala, se realiza hemostasia por compresión. El paciente cursa internación en sala por 24 horas. Si no existen complicaciones se otorga alta hospitalaria y el paciente continúa seguimiento por médico de cabecera.

\section{RESULTADOS}

En el período comprendido entre los meses de junio de 2017 y junio de 2018 se realizó embolización a 6 pacientes con síndrome de intolerancia post fallo de trasplante renal. De los pacientes, 5 eran mujeres y uno hombre. La edad promedio 38 años. Todos en tratamiento hemodialítico. El tiempo Uno 


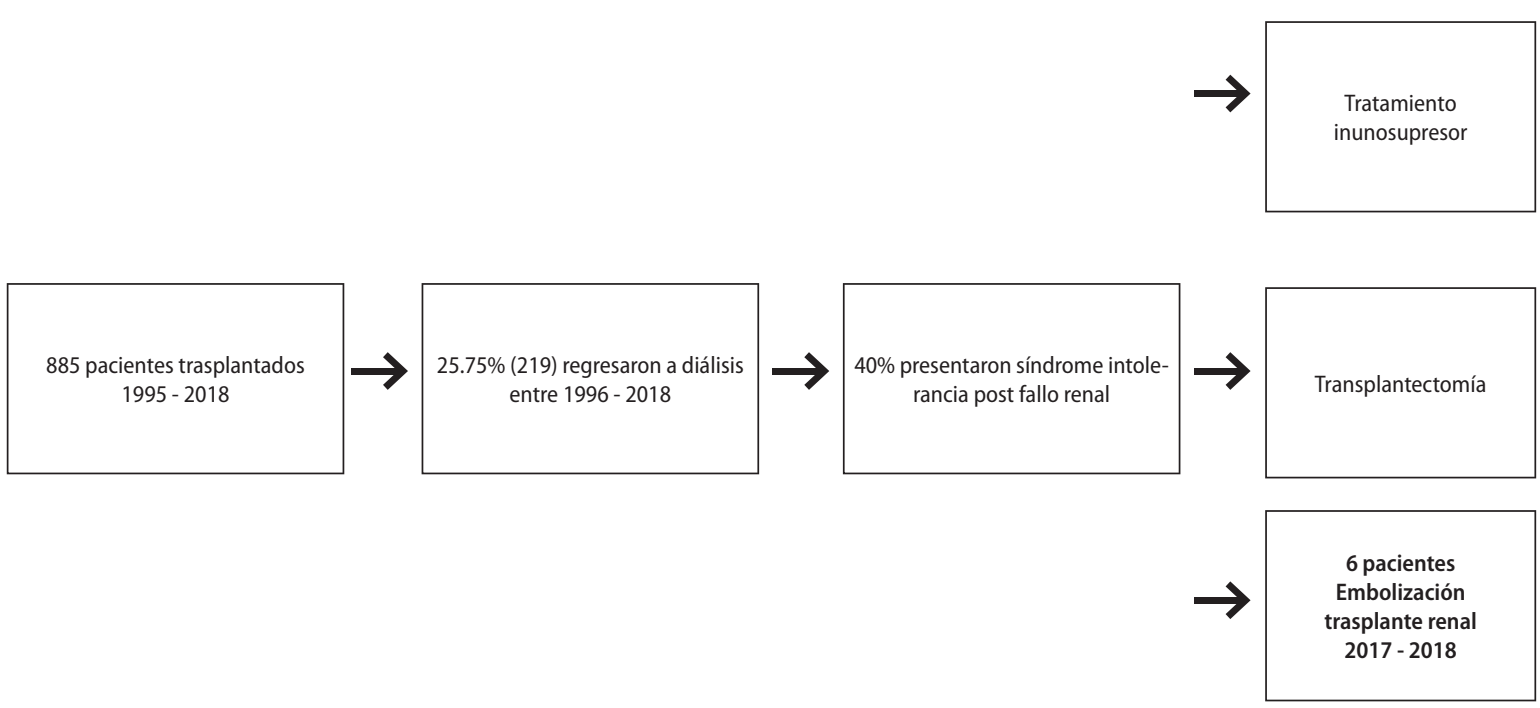

Figura 1.

TABLA 1.

\begin{tabular}{|l|c|}
\hline Edad promedio & $38 \pm 18$ años \\
\hline Hombres & 1 caso \\
\hline Mujeres & 5 casos \\
\hline Tiempo inicio diálisis/embolización promedio & 8 meses \\
\hline Fiebre y hematuria & 3 casos \\
\hline Dolor del injerto y anemia & 2 casos \\
\hline Dolor del injerto & 1 caso \\
\hline Duración de la internación & 1 día \\
\hline
\end{tabular}

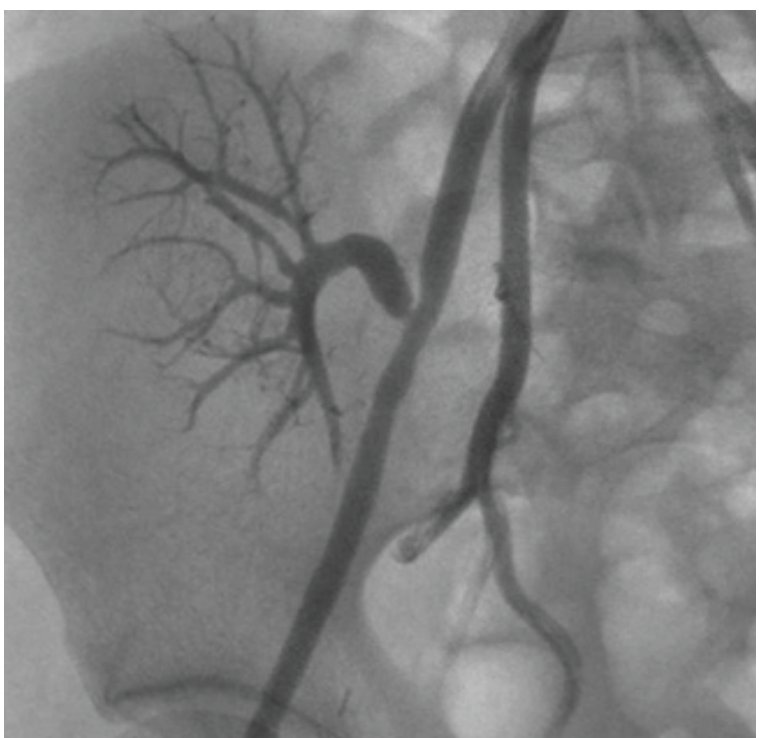

FB E preembolización.

promedio entre el inicio de diálisis y la embolización fue de 8 meses. Los síntomas fueron fiebre y hematuria (3 casos), dolor del injerto y anemia (2 casos), dolor del injerto aislado (1 caso). Previo a la realización de la embolización se descartaron infección y la presencia de tumores en el injerto (urocultivo y ecografía). En todos los casos se realizó profilaxis antibiótica. Los pacientes se internaron el día del procedimiento y fueron dados de alta al día siguiente (Tabla 1).

Se utilizó la vía femoral homolateral en todos los casos. Se administraron 5000 UI de heparina sódica. Se cateterizó selectivamente arteria renal del injerto con catéter $5 \mathrm{Fr}$. Se
TABLA 2.

\begin{tabular}{|l|c|}
\hline Duración del procedimiento promedio & 30 minutos \\
\hline Volumen de contraste promedio & $75 \mathrm{ml}$ \\
\hline 1 jeringa prellenada & 5 casos \\
\hline 2 jeringas prellenadas & 1 caso \\
\hline Uso de coils & 4 casos \\
\hline Éxito angiográfico & $100 \%$ \\
\hline Síndrome posembolización & 1 caso \\
\hline
\end{tabular}

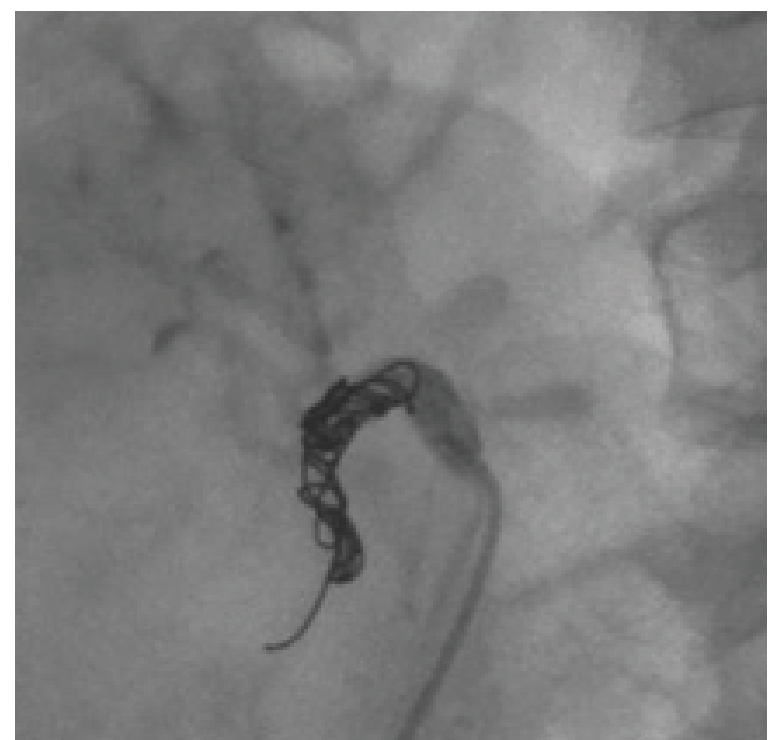

FB E posembolización.

realizó angiografía selectiva. Para la embolización se utilizaron microesferas de polivinilo de 300-500 $\mu \mathrm{m}$; en 5 casos fue suficiente con una jeringa prellenada. En uno de los casos, debido al tamaño del injerto, fueron necesarias dos jeringas prellenadas. En 4 casos se completó embolización de arteria renal con coils. El éxito angiográfico se define como la obliteración completa de la circulación del injerto. Fue alcanzado en todos los casos. La duración promedio del procedimiento fue de 30 minutos. El volumen de contraste utilizado fue menor a los $75 \mathrm{ml}$ en todos los casos (Tabla 2). 

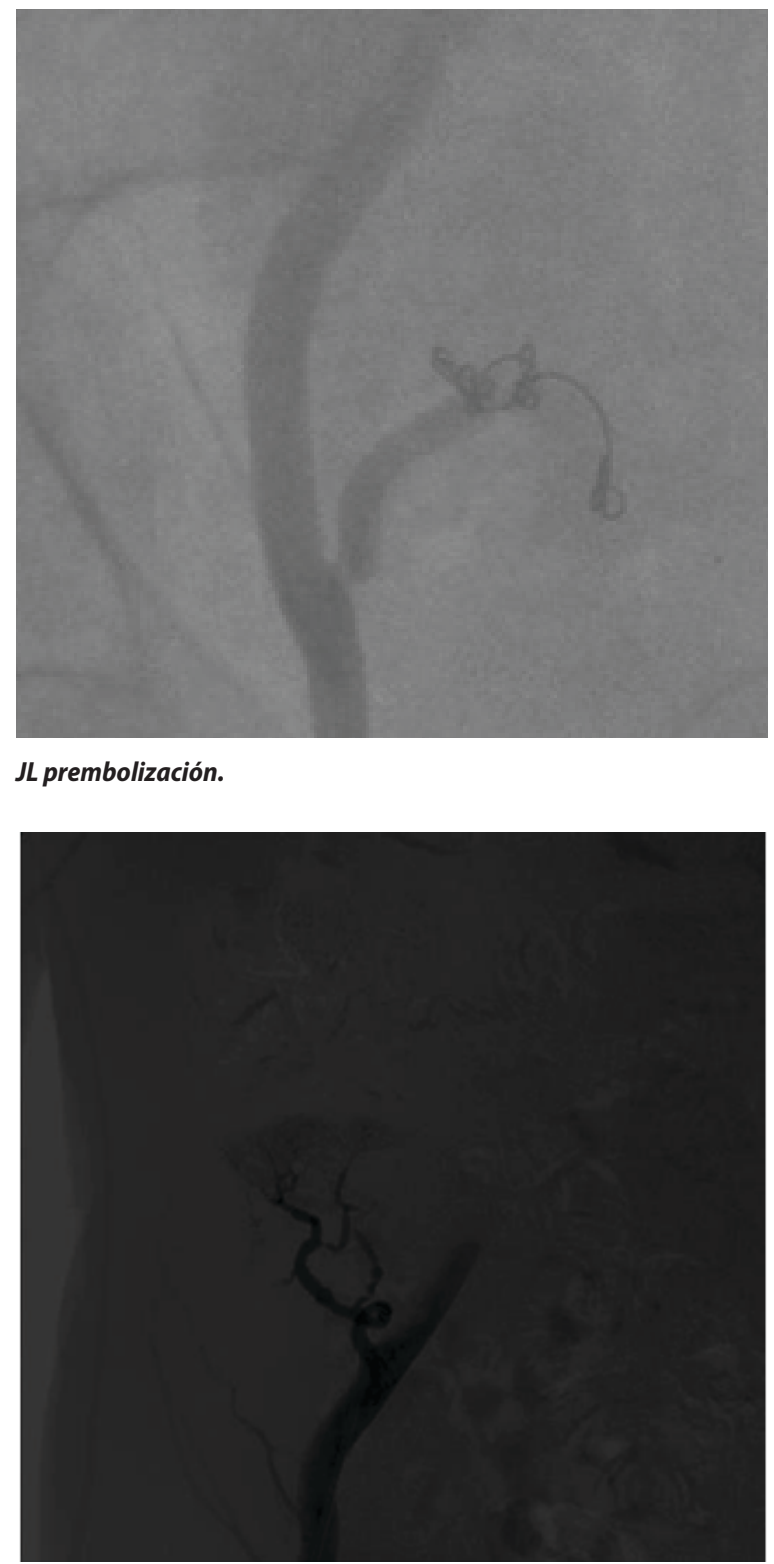

VL preembolización.

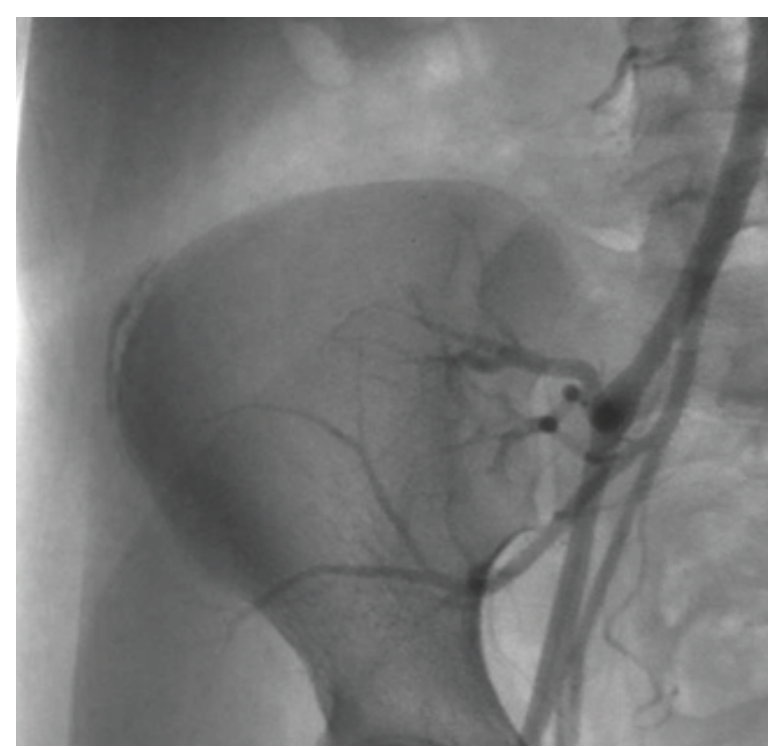

SV preembolización.
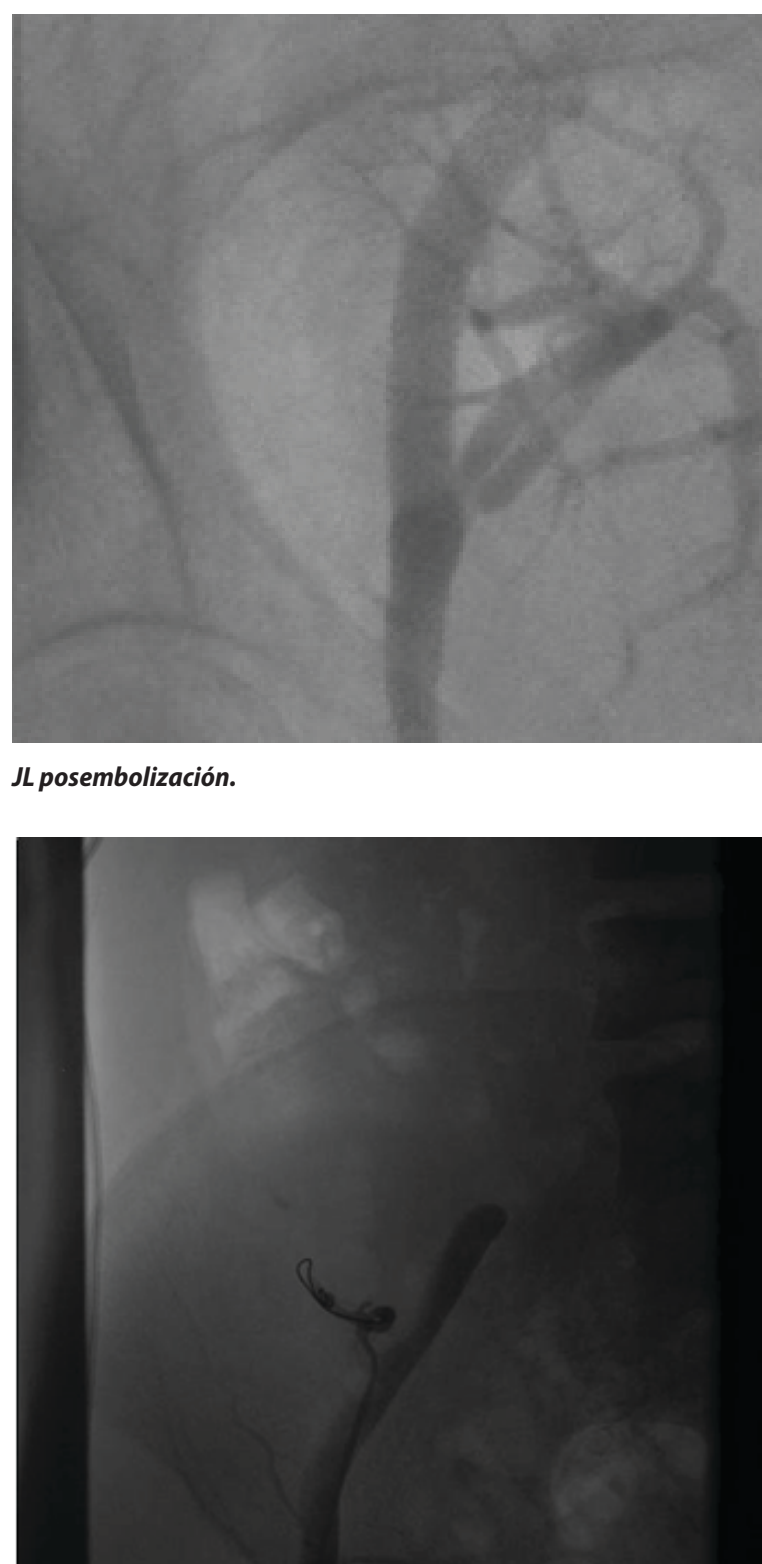

VL posembolización.

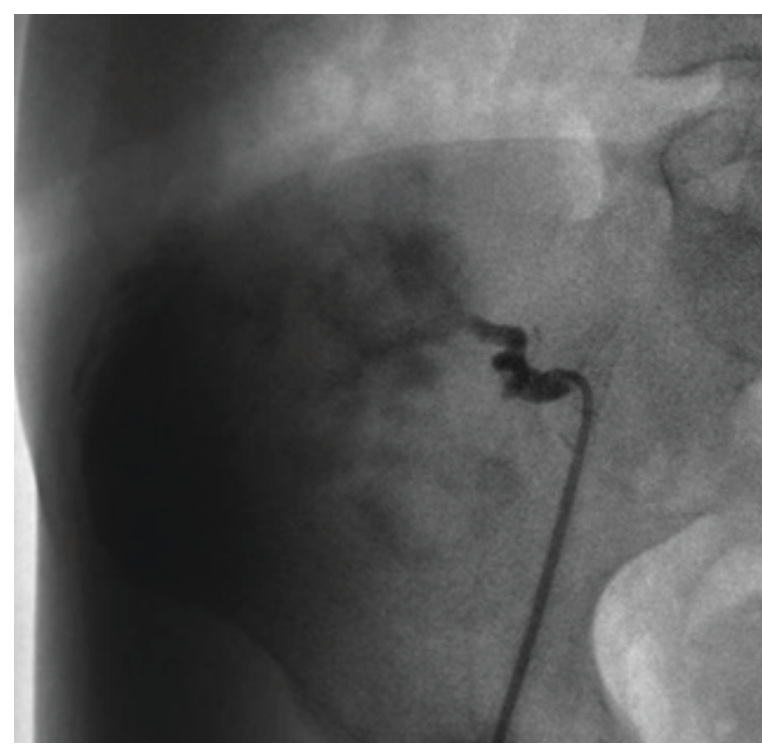

SV posembolización. 


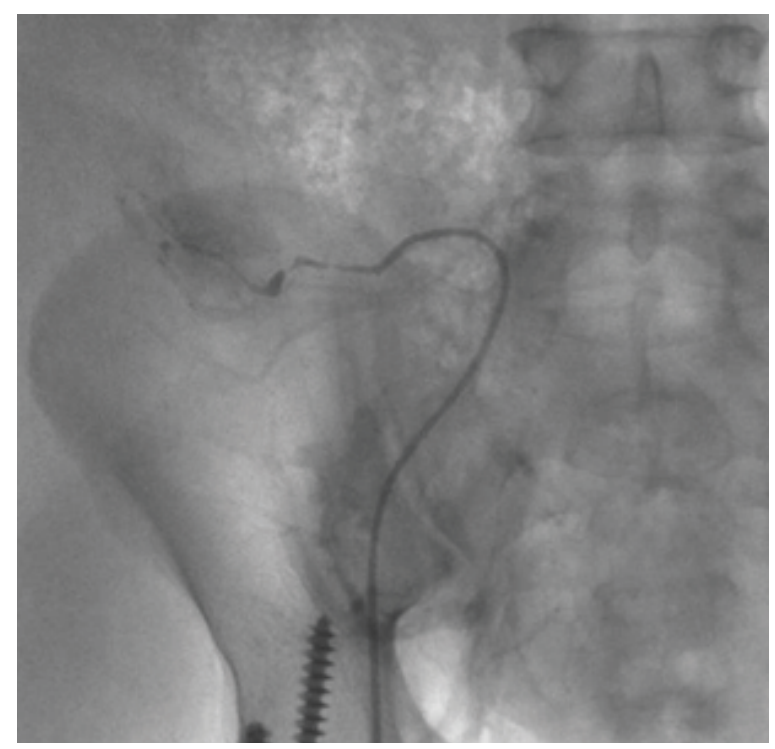

DG preembolización.

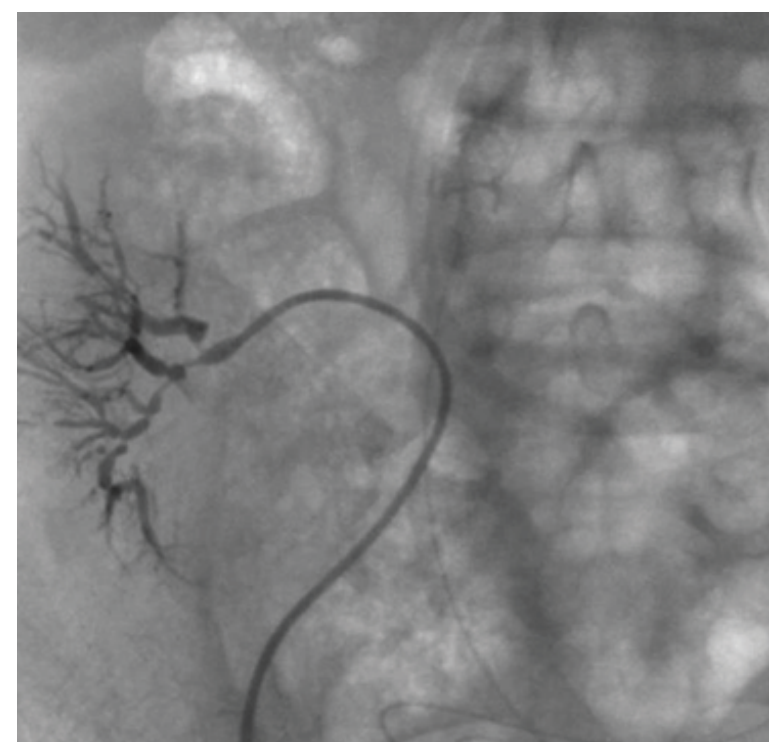

\section{GV preembolización.}

de los pacientes presentó síndrome posembolización caracterizado por fiebre 12 horas luego del procedimiento. Fue tratado con antiinflamatorios no esteroideos, con buena respuesta. Todos los pacientes presentaron remisión completa de los síntomas y ausencia de recurrencia durante el período de seguimiento.

\section{DISCUSIÓN}

Hasta un $40 \%$ de los pacientes que vuelven a diálisis tras un trasplante renal fallido desarrollan intolerancia inmunológica al injerto cuando se suspende o disminuye la inmunosupresión. La reintroducción de la terapia inmunosupresora conlleva elevado riesgo de infecciones, neoplasias y aumento del riesgo cardiovascular.

Este síndrome se manifiesta por la presencia de febrícula, malestar general, astenia, hematuria, dolor o incremento del tamaño del injerto ${ }^{1}$. Existen formas de presentación menos evidentes, como cifras elevadas de PCR y más bajas de albúmina y prealbúmina o requerimiento de altas dosis de eritropoyeti$\mathrm{na}^{5}$. El tratamiento tradicional es la ablación quirúrgica del in-

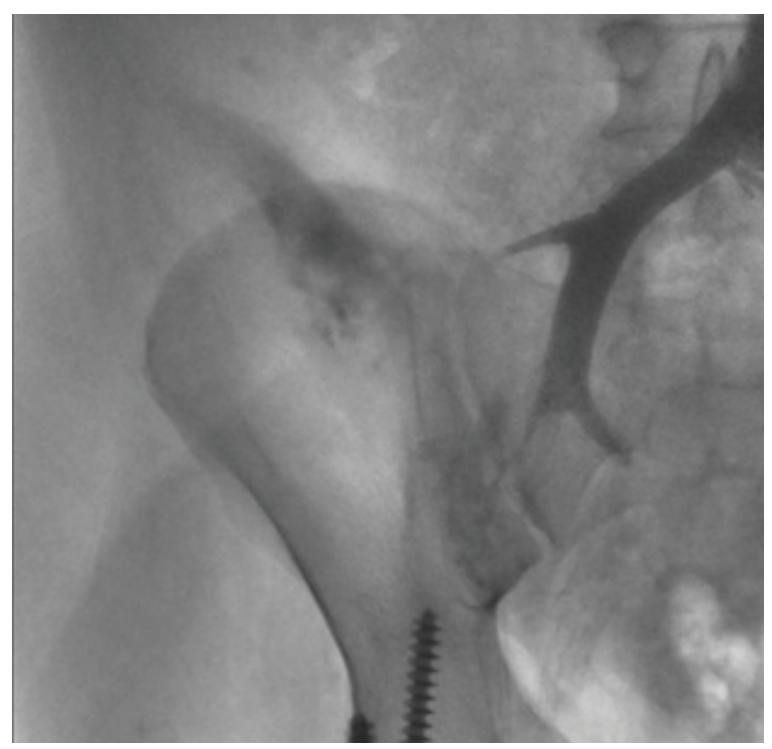

\section{DG posembolización.}

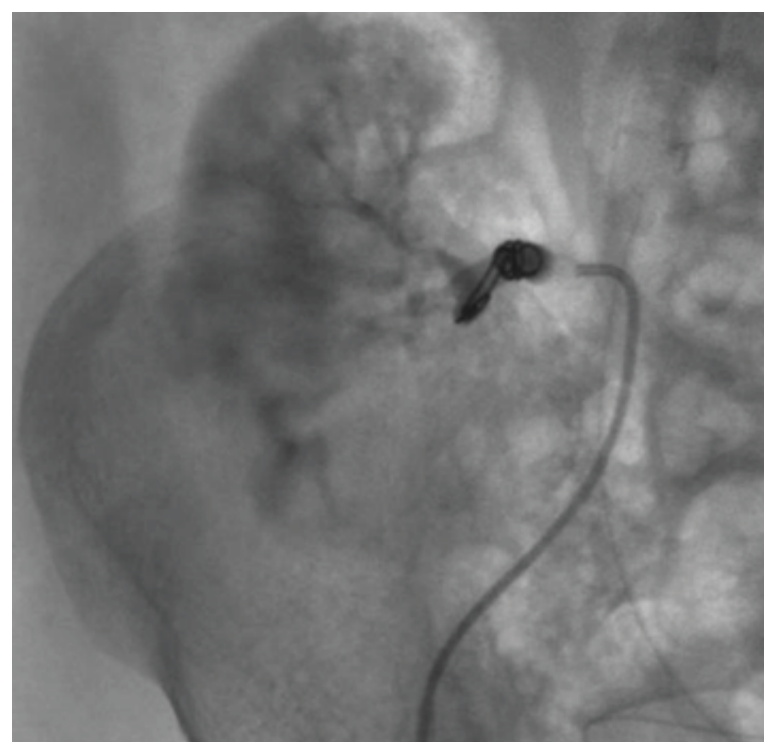

\section{GV posembolización.}

jerto. Este procedimiento presenta elevada morbimortalidad debido a las comorbilidades de los pacientes y a las dificultades técnicas para la extracción del injerto que frecuentemente presenta importantes adherencias a los tejidos circundantes. A pesar de un descenso de la morbimortalidad de la trasplantectomía en los últimos años, el riesgo de complicaciones graves continúa siendo cercano al 10\%, siendo las complicaciones más frecuentes las infecciosas y el sangrado ${ }^{4}$. La embolización arterial con microesferas seguida de la inserción de coils metálicos es un procedimiento sencillo, seguro y eficaz; y constituye una alternativa a la nefrectomía del injerto. Sin embargo, estaría contraindicada en casos de infección sobreañadida, presencia de neoformaciones o alto riesgo de rotura del injerto por complicaciones quirúrgicas o rechazo grave intratable ${ }^{6}$. Por este motivo es de fundamental importancia la evaluación previa de los pacientes con ecografía del injerto y urocultivo. La principal complicación de la embolización es el llamado síndrome posembolización, caracterizado por fiebre y dolor del injerto, que habitualmente cede luego de 48-72 hs. En las series publicadas esta complicación afecta aproximadamente al 60\% de los pacientes, en nuestro caso se presentó en solo uno de ellos. En la mayoría de 
los casos responde al tratamiento con antiinflamatorios no esteroideos. En caso de no remisión de los síntomas, se debe sospechar falla de la embolización?

\section{CONCLUSIÓN}

Debido al incremento de los pacientes que reciben trasplante renal, es de esperar un aumento en la incidencia del síndrome de intolerancia tras fallo del injerto. Dado que la embolización del injerto renal no funcionante constituye un tratamiento sencillo, seguro y eficaz de este síndrome, es importante para los hemodinamistas de hospitales en los que se realiza trasplante renal conocer sus indicaciones y las características del procedimiento.

\section{BIBLIOGRAFÍA}

1. Schoolwerth AC, Engelgau MM, Hostetter TH, et al. Chronic kidney disease: a public health problem that needs a public health action plan. Prev Chronic Dis 2006;3:A57

2. Verresen L, Vanrenterghem Y, Waer M, Hauglustaine D, Michielsen P. Corticosteroid withdrawal syndrome in dialysis patients. Nephrol Dial Transplant 1988:3:476-7.

3. Kiberd BA, Belitsky P. The fate of the failed renal transplant. Transplantation 1995;59:645-7.

4. Vanrenterghem Y, Khamis S. The management of the failed renal allograft. Nephrol Dial Transplant 1996;11:955-7

5. López-Gómez JM, Pérez-Flores I, Jofre R, et al. Presence of a failed kidney transplant in patients who are on hemodialysis is associated with chronic inflammatory state and erythropoietin resistance. J Am Soc Nephrol 2004:15:2494-501

6. Isabel Pérez-Flores, Ana Sánchez-Fructuos, Roberto Marcén, Ana Fernández, Milagros Fernández Lucas, José Luis Teruel. Manejo del injerto renal fallido. Nefrectomía versus embolización. Nefrología 2009;29(Sup. 1):54-61.

7. Henrique Mochida Takase, Mariana Moraes Contti, Hong Si Nga, Ariane Moyses Bravin, Mariana Farina Valiatti, Regina Paolucci El-Dib, Luis Gustavo Modellide Andrade. Nephrectomy Versus Embolization of Non-Functioning Renal Graft: A Systematic Review with a Proportional Meta-Analysis.Ann Transplant 2018; 23:207-217.1

8. Capocasale E, Larini P, Mazzoni MP et al: Percutaneous renal artery embolization of nonfunctioning allograft: Preliminary experience. Transplant Proc, 2005; 37: 2523-2

9. Cofan F, Real MI, Vilardell J et al: Percutaneous renal artery embolisation of non-functioning renal allografts with clinical intolerance. Transpl Int 2002; 15: 149-55

10. Gonzalez-Satue C, Riera L, Franco E et al: Percutaneous embolization of nonfunctioning renal graft as therapeutic alternative to surgical transplantectomy. Actas Urol Esp, 2000; 24: 319-24

11. Delgado P, Diaz F, Gonzalez A et al: Intolerance syndrome in failed renal allografts: Incidence and efficacy of percutaneous embolization. Am J Kidney Dis, 2005; 46: 339-44 